\title{
Interface Profile of the Ising Ferromagnet in Two Dimensions
}

\author{
D. B. Abraham^
}

Laboratoire de Physique Théorique, Ecole Polytechnique Fédérale, Lausanne, Switzerland

P. Reed

Department of Mathematics, University of Manchester, Manchester, England

\begin{abstract}
The interface profile of the two-dimensional Ising ferromagnet is obtained for all temperatures in the thermodynamic limit. The width of the interface depends on its length as (length) $)^{1 / 2}$.
\end{abstract}

\section{Introduction}

Recently considerable effort has been devoted to the unravelling of the phenomenon of phase separation in the Ising ferromagnet or in the equivalent lattice gas $[1,2,3]$. This paper reports on exact calculation of the surface tension and interface profile for the two dimensional case with nearest neighbour interactions and zero "gravitational" field [0]. First we shall review briefly exact results already established and then we shall relate our computation to them.

\section{Notation and Results}

Let $\Lambda$ be a crystal lattice in $d$ dimensions with unit edges; it may therefore be regarded as a subset of $\mathbb{Z}^{d}$. At each vertex $i$ let there be a spin $\sigma_{i}= \pm 1$. The energy of a spin configuration $\{\sigma\}$ is given by:

$$
E_{\Lambda}\{\sigma\}=-J \sum_{\langle i, j\rangle \subset \Lambda} \sigma_{i} \sigma_{j}-\mathscr{B}_{\Lambda}\{\sigma\}
$$

with associated probability measure

$$
p_{A, \mathscr{P}}(\{\sigma\})=Z^{-1} \exp -\beta E_{\Lambda}(\{\sigma\}) \text {. }
$$

The sum in (2.1) is over nearest neighbour pairs on $\Lambda$ and $\mathscr{B}_{\Lambda}(\{\sigma\})$ is a boundary term [1], examples of which will be encountered later.

Let $\sigma_{A}$ be defined for $A \subset \mathbb{Z}^{d}$ by

$$
\sigma_{A}=\prod_{i \in A} \sigma_{i}
$$

* On leave from Oxford University. Partially supported by NSF grant no. MPS 7520638. 
where $i$ is a vertex of $\mathbb{Z}^{d}$, and let its expectation with respect to (2.2) be denoted $\left\langle\sigma_{A}\right\rangle_{A, \mathscr{B}_{A}}$. Then the state is the collection of all limits:

$$
\left\langle\sigma_{A}\right\rangle_{\mathscr{B}}=\lim _{\Lambda \rightarrow \infty}\left\langle\sigma_{A}\right\rangle_{\Lambda, \mathscr{B}_{A}}
$$

where the limit is of van Hove type. No magnetic field term is included in (2.1) since only then is there a possible lack of uniqueness of the state [6]; this possibility is indeed realized. For instance, the spontaneous magnetization $m^{*}$ is given by:

$$
m^{*}=\left\langle\sigma_{p}\right\rangle_{+}=-\left\langle\sigma_{p}\right\rangle_{-}
$$

where the boundary condition means that $\sigma_{i}=+1 \forall i \in \partial \Lambda, \forall \Lambda$. It is known [4] that for $d=2, m^{*}=\left(1-(\sinh 2 K)^{-4}\right)^{1 / 8}$, so that $m^{*}>0 \forall K>K_{c}$ where $K=\beta J$ and $\sinh 2 K_{c}=1 ; \beta_{c}^{-1}$ may be defined as the critical temperature for the model.

The states $\left\langle\sigma_{A}\right\rangle_{ \pm}$have a special significance, as shown in a recent distillation of the quintescence of duality by Messager and Miracle-Sole [5]. Provided one considers only translationally invariant states, then

$$
\left\langle\sigma_{A}\right\rangle_{\mathscr{B}}=\lambda\left\langle\sigma_{A}\right\rangle_{+}+(1-\lambda)\left\langle\sigma_{A}\right\rangle_{-}
$$

where $0 \leqq \lambda \leqq 1$, and $\lambda$ is determined by $\mathscr{B}$. Thus $\left\langle\sigma_{A}\right\rangle_{ \pm}$may be considered as describing pure phases [6].

It is now clear how to generate an interface between phases: on a $(2 M+1) \times$ $(2 N+1)$ rectangular lattice with vertex coordinates $(i, j)-M \leqq i \leqq M,-N \leqq j \leqq N$, consider the boundary condition $B_{A}^{+-}$specified by $\sigma_{i j}=+1$ (resp. -1 ) if $i \geqq 0$ (resp. $i<0)(i, j) \in \partial \Lambda$. The interface profile is defined by

$$
\left\langle\sigma_{p}\right\rangle_{N}=\lim _{M \rightarrow \infty}\left\langle\sigma_{p}\right\rangle_{\Lambda, \mathscr{B}_{\Lambda}^{+}}
$$

and the surface tension is

$$
\tau=\lim _{N \rightarrow \infty} \frac{1}{2 N+1} \lim _{M \rightarrow \infty} \log Z_{\Lambda}^{+-} / Z_{\Lambda}^{+}
$$

where

$$
Z_{\Lambda}^{+-}=\sum_{\{\sigma\}} \exp -\beta E_{\Lambda, \mathscr{B}_{\Lambda}^{+-}}(\{\sigma\})
$$

and $Z_{\Lambda}^{+}$is defined analogously with boundary condition $\mathscr{B}_{\Lambda}^{+}: \sigma_{i}=+1 \forall i \in \partial \Lambda$.

The surface tension $\tau$ has already been obtained using the theory of the transfer matrix of a pure phase [7].

Its value is:

$$
\begin{array}{cl}
\tau=2 K+\log \tanh K & K>K_{c} \\
0 & K \leqq K_{c} .
\end{array}
$$

This is precisely Onsager's result calculated according to a quite different definition [8]. Yet others, due to Camp and Fisher [2], to Fisher and Ferdinand [9] and to Gallavotti and Martin-Löf $[10,3,21]$ give the same result and the agreement is no coincidence; but we shall not pursue it further here. Rather, we return to (2.7), the interface profile, and a related problem.

Contours on the dual lattice $\Lambda^{*}$ may be constructed by drawing line segments between antiparallel spin pairs on $\Lambda$; this is described carefully in Ref. [10]. The only contour configurations which contribute are those for which 0,2 , or 4 edges meet at any vertex inside $\Lambda^{*}$. There is a long contour $\lambda$ which intersects $\partial \Lambda$ at opposite sides. Gallavotti [11] proved an interesting theorem about $\lambda$ : 
Theorem 1 (Gallavotti). Let $Q$ be any finite region in $\mathbb{Z}^{2}$. If $\beta$ is large enough there exists a function $D(N)$ such that

$$
\lim _{N \rightarrow \infty} P(\{d(\lambda, Q)>D(N)\})=1
$$

where $P(X)$ is the probability of event $X$ with respect to the measure (2.2) with the boundary condition $\mathscr{B}_{\Lambda}^{+-}$above, as $M \rightarrow \infty$. The "best" choice of the function $D$ is $D(N) \sim N^{1 / 2}$.

Because the long contour $\lambda$ is highly corrugated there is no immediate connection between Theorem 1 and the profile (2.7). Indeed, the profile might still be sharp, and furthermore, it might sharpen on raising the temperature because the degree of corrugated might well increase. But the following theorem, which is the main result of this paper, shows that this is not in fact the case.

Theorem 2. Let $p=\alpha N^{\delta}, 0 \leqq \delta$ and let $T>0:$ then

$$
\begin{aligned}
& \lim _{N \rightarrow \infty}\left\langle\sigma_{p}\right\rangle_{N}=\left\{\begin{array}{l}
0, \delta<\frac{1}{2} \\
m^{*} \operatorname{sgn} \alpha, \delta>\frac{1}{2}
\end{array}\right. \\
& \lim _{N \rightarrow \infty}\left\langle\sigma_{\alpha N^{1 / 2}}\right\rangle_{N}=m^{*} \operatorname{sgn} \alpha \Phi(b|\alpha|)
\end{aligned}
$$

where

$$
b=\left(\sinh 2\left(K-K^{*}\right)\right)^{1 / 2}, e^{-2 K^{*}}=\tanh K
$$

and

$$
\Phi(x)=\frac{2}{\sqrt{\pi}} \int_{0}^{x} e^{-u^{2}} d u .
$$

The function $\Phi(x)$ has the properties:

$$
\text { (i) } \Phi(x)=\frac{2}{\sqrt{\pi}} \sum_{0}^{\infty} \frac{(-1)^{j}}{j !} \frac{x^{2 j+1}}{2 j+1}
$$

hence $\Phi(x)$ is analytic near $x=0$. In fact, $\Phi(x)$ is entire; it behaves near $x=\infty$ as:

$$
\Phi(x) \sim 1-\frac{e^{-x^{2}}}{x} \sum_{0}^{\infty}(-1)^{r} x^{-2 r} \Gamma\left(r+\frac{1}{2}\right) .
$$

Remarks. 1. The result can be generalized to ours finite number of spin reversals on the boundary as $M \rightarrow \infty$; then $\left\langle\sigma_{p}\right\rangle_{\mathscr{B}}$ is independent of $p$, though of course not necessarily zero. This strengthens the conjecture that there are no translationally non-invariant equilibrium states for $d=2$. Messager [12] has established an interesting connection between $\mathscr{B}_{A}^{+-}$and a class of general ones which reinforces the above remark. For the analogous $\mathscr{B}_{A}^{+-}$when $d=3$, Dobrushin [13] has shown that $\left.\left\langle\sigma_{p}\right\rangle\right\rangle\left\langle\sigma_{p-1}\right\rangle$ for $T\left\langle T^{\prime}\left(T^{\prime} \sim \cdot 2^{-32} T_{c}\right.\right.$ !). The range of validity of this result was extended to $T_{c}(3)>T_{c}(2) \geqq T^{\prime}$ by van Beijeren [14]. There is evidence that the profile for $d=3$ becomes diffuse for $T>T_{c}(2)$ [15].

2. The dividing point $\delta=\frac{1}{2}$ is uniform in $T$. Moreover, the function $\lim \left\langle\sigma_{\alpha N^{1 / 2}}\right\rangle_{N}$ is analytic in $T$ for any $\alpha$ provided $T \neq 0, T_{c}$. At $T=T_{c}$ there is a square root branch point.

3. Consider a contour $C$ on $\mathbb{Z}^{2}$ from $(-N, 0)$ to $(N, 0)$ which moves only up or down or to the right at each vertex, starting from $(-N, 0)$. The statistical 
weight is:

$$
p_{N}(C)=Z_{N}^{-1} \exp -2 K^{l(C)}
$$

where $l(C)$ is the Euclidean length on $\mathbb{Z}^{2}$.

Let the intercept of $C$ on the line $(0, y)$ be $y(C)$. Define the magnetization profile of the ensemble of all $C$ by:

$$
m_{N}(t)=m^{*} \sum_{C}(P(y(C) \geqq t)-P(y(C)<t)) .
$$

Standard random walk arguments, for instance using the central limit theorem, shwo that if $t=\alpha N^{\delta}$ then

$$
\begin{aligned}
& \lim _{N \rightarrow \infty} m_{N}(t)=\left\{\begin{array}{l}
0, \delta<\frac{1}{2} \\
m^{*} \operatorname{sgn} \alpha, \delta>\frac{1}{2}
\end{array}\right. \\
& \lim _{N \rightarrow \infty} m_{N}\left(\alpha N^{1 / 2}\right)=m^{*} \operatorname{sgn} \alpha \Phi\left(2^{1 / 2}|\alpha| \sinh K\right)
\end{aligned}
$$

and

The origin of the Gaussian distribution is rather clear in this case. This model is the Temperley string [16].

Further, the incremental free energy is:

$$
\lim _{N \rightarrow \infty}(2 N)^{-1} \log Z_{N}=\left\{2\left(K-K^{*}\right)\right. \text {. }
$$

At sufficiently low temperature, Theorem 2 is recaptured precisely, including the scaling factor $b$ in the argument of $\Phi$. This is highly significant: the long contour $\lambda$ and the remaining contours (which are relatively short [1]) behave essentially independently. One wonders whether this might be true for $d=3$ and what bearing it might have on Remark 1 above.

4. This theory contrasts sharply with that of Fisk and Widom [22] in which the free energy density is a functional of the density profile. Even with rescaled lengths with $\delta=\frac{1}{2}$ there is no agreement. One wonders whether this basic theory would be recaptured with a small gravitational field.

The rest of this paper is devoted to the derivation of Theorem 2.

\section{Proof of Main Theorem}

First we relate the problem to one with more symmetry by a clustering argument. Consider a right cylindrical lattice $\Lambda$ with $M$ rows and $2 N+1$ columns. Let the boundary condition $\mathscr{B}_{\Lambda}(s)$ be defined by $\sigma_{i, \pm N}=1$ (resp. -1 ) for $1 \leqq i \leqq S$ (resp. $s<i \leqq M)$. The interface profile is defined in terms of $\mathscr{B}_{A}^{+-}$by (2.7). Then we have:

Lemma 1. The limit exists and

$$
\left\langle\sigma_{p}\right\rangle_{N}=\lim _{s \rightarrow \infty} \lim _{M \rightarrow \infty}\left\langle\sigma_{(p, 0)}\right\rangle_{\mathscr{B}_{\Lambda}(s)} .
$$

The surface tension is:

$$
\tau=\lim _{N \rightarrow \infty} \frac{1}{4 N} \lim _{s \rightarrow \infty} \lim _{M \rightarrow \infty} \log Z\left(\mathscr{B}_{\Lambda}(s)\right) .
$$

Proof. Here we use the transfer matrix which acts in a direction perpendicular to the cylinder axis [7]. The details are given in Appendix A.

By applying the FKG inequalities [11] to the boundary condition, we obtain: 
Lemma 2. i) $\left\langle\sigma_{p}\right\rangle_{\mathscr{B}_{A}^{+}} \geqq\left\langle\sigma_{p-1}\right\rangle_{\mathscr{B}_{A}^{+}}-\forall \Lambda$.

Using the limit part of Lemma 1 we have:

$$
\left\langle\sigma_{p}\right\rangle_{N} \geqq\left\langle\sigma_{p-1}\right\rangle_{N} .
$$

ii) $\left|\limsup _{N \rightarrow \infty} \lim _{M \rightarrow \infty}\left\langle\sigma_{p}\right\rangle_{\mathscr{B}_{\Lambda}^{+}}\right| \leqq m^{*}$.

We shall now use the transfer matrix along the axis of symmetry of the cylindrical lattice $\Lambda$ with boundary condition $\mathscr{B}_{\Lambda}(s)$. Let

$$
V_{1}=\exp -K^{*} \sum_{1}^{M} \sigma_{j}^{z} \quad V_{2}=\exp K \sum_{1}^{M} \sigma_{j}^{x} \sigma_{j+1}^{x}
$$

with

$$
\exp -2 K^{*}=\tanh K
$$

and let $| \pm\rangle$ be the states defined by:

$$
\sigma_{j}^{x}| \pm\rangle= \pm| \pm\rangle, 1 \leqq j \leqq M .
$$

Then standard techniques yield the results for the surface tension and interface profile:

$$
\tau=\lim _{N \rightarrow \infty} \frac{1}{4 N} \lim _{s \rightarrow \infty} \lim _{M \rightarrow \infty} \log \frac{\left\langle-\left|R(s)\left(V_{2} V_{1}\right) V_{2} R(s)\right|-\right\rangle}{\left\langle-1\left(V_{2} V_{1}\right)^{2 N} V_{2} \mid-\right\rangle}
$$

and

$$
\left\langle\sigma_{p}\right\rangle_{N}=\lim _{s \rightarrow \infty} \lim _{M \rightarrow \infty} \frac{\left\langle-\left|R(s)\left(V_{2} V_{1}\right)^{N} \sigma_{p}^{x}\left(V_{2} V_{1}\right)^{N} V_{2} R(s)\right|-\right\rangle}{\left\langle-1 R(s)\left(V_{2} V_{1}\right)^{2 N} V_{2} R(s) \mid-\right\rangle}
$$

where $R(s)$ reverses all spins for 1 to $s$ inclusive in the state $|-\rangle$.

Clearly

$$
V_{2}| \pm\rangle=e^{M K}| \pm\rangle
$$

and

$$
V_{2} R(s)=R(s) V_{2} e^{-2 K}
$$

so that (3.8) and (3.9) may be expressed in terms of the symmetrized transfer matrix $V=V_{2}^{1 / 2} V_{1} V_{2}^{1 / 2}$ through the quantities:

$$
\begin{aligned}
Z_{M}^{+-}(s) & =\left\langle-\left|R(s) V^{2 N} R(s)\right|-\right\rangle \\
Z_{M}^{+} & =\left\langle-\left|V^{2 N}\right|-\right\rangle
\end{aligned}
$$

and

$$
\Sigma_{M}^{+-}(s \mid p)=\left\langle-\left|R(s) V^{N} \sigma_{p}^{x} V^{N} R(s)\right|-\right\rangle .
$$

Evidently

and

$$
\tau=\lim _{N \rightarrow \infty}(4 N)^{-1} \lim _{s \rightarrow \infty} \lim _{M \rightarrow \infty} \log Z_{M}^{+-}(s) / Z_{M}^{+}
$$

$$
\left\langle\sigma_{p}\right\rangle_{N}=\lim _{s \rightarrow \infty} \lim _{M \rightarrow \infty} \Sigma_{M}^{+-}(s \mid p) / Z_{M}^{+-}(s) .
$$

The strategy of the remainder of the calculation is to recognize, if necessary with hind sight, that $| \pm\rangle$ and $R(s)$ have very amenable expressions in the spinor algebra in terms of which the diagonalization is expressed [19]. Thus it is natural 
to spectrally decompose $V$ in (3.12) and (3.13). The notation of Ref. 19 will be used throughout the remainder.

The collections of eigenvectors of $V$ with $j$ particles which lie in $\mathfrak{S}_{M}^{ \pm}$will be denoted $\mathscr{B}_{j}^{ \pm}$. Let $\mathscr{P}_{2}$ be the projector onto the orthogonal complement of $\mathscr{B}_{0}^{+} \cup \mathscr{B}_{0}^{-} \cup \mathscr{B}_{2}^{+} \cup \mathscr{B}_{2}^{-}$. Then

$$
\begin{aligned}
\Sigma_{M}^{+-}(s \mid p)= & \sum_{j, k \in \mathscr{B}_{0}^{ \pm} \cup \mathscr{B}_{2}^{ \pm}} \Lambda_{j}^{N} \Lambda_{k}^{N}\langle-|R(s)| j\rangle\left\langle j\left|\sigma_{p}^{x}\right| k\right\rangle\langle k|R(s)|-\rangle \\
& +\left\langle-\left|R(s) \mathscr{P}_{2} V^{N} \sigma_{p}^{x} V^{N} \mathscr{P}_{2} R(s)\right|-\right\rangle .
\end{aligned}
$$

The following bound

$$
\begin{aligned}
& \left|\left\langle-\left|R(s) \mathscr{P}_{2} V^{N} \sigma_{p}^{x} V^{N} \mathscr{P}_{2} R(s)\right|-\right\rangle\right| \\
& \leqq\left\langle-\left|R(s) \mathscr{P}_{2} V^{2 N} R(s)\right|-\right\rangle
\end{aligned}
$$

is readily obtained, relating to the analogous problem for $Z_{M}^{+{ }^{-}}(s)$ which produces precisely the term on the rhs of (3.18). This is bounded by obtaining $Z_{M}^{+-}(s)$ in closed form using the method of Schultz et al. [20], and then subtracting the contribution from $\mathscr{B}_{0} \cup \mathscr{B}_{2}$ (see Appendix A); the term from $\mathscr{B}_{2}$ only survives the limits in (3.15) and (3.16).

The construction of $| \pm\rangle[21]$ and $R(s)$ follows: from (3.7),

$$
| \pm\rangle=\prod_{1}^{M} 2^{-1 / 2}\left(1+\sigma_{j}^{x}\right)|0\rangle
$$

where $\sigma_{j}^{z}|0\rangle=-|0\rangle, \forall j=1, \ldots, M$. But the maximum eigenvalue of $V$ when $K^{*}=0$ is doubly degenerate with all spins parallel. Let the normalized eigenvectors be $\left|\Phi_{ \pm}^{0}\right\rangle$, in $\mathfrak{H}_{M}^{ \pm}$. Now from (3.19), $P|+\rangle=|-\rangle$. Thus:

$$
\left|\Phi_{ \pm}^{0}\right\rangle=2^{-1 / 2} a_{ \pm}(|+\rangle \pm|-\rangle),\left|a_{ \pm}\right|=1
$$

satisfying $P\left|\Phi_{ \pm}^{0}\right\rangle= \pm\left|\Phi_{ \pm}^{0}\right\rangle$. Inverting (3.20) gives:

$$
| \pm\rangle=2^{-1 / 2}\left(a_{+}^{*}\left|\Phi_{+}^{0}\right\rangle \pm a^{*}\left|\Phi_{-}^{0}\right\rangle\right) \text {. }
$$

Now from Ref. [19] Equations (2.37), (2.39), (2.40)

$$
\left|\Phi_{+}^{0}\right\rangle=\left|\hat{\Phi}_{+}\right\rangle,\left|\Phi_{-}^{0}\right\rangle=F_{0}^{+}\left|\hat{\Phi}_{-}\right\rangle
$$

where

$$
\left|\hat{\Phi}_{ \pm}\right\rangle=\prod_{0<k_{ \pm}<\pi}\left(-\sin \left(k_{ \pm} / 2\right)+i \cos \left(k_{ \pm} / 2\right) F_{-k_{ \pm}}^{\dagger} F_{k_{ \pm}}^{\dagger}\right)|0\rangle
$$

with $\exp i M k_{ \pm}=\mp 1$, and $M$ is always taken even. Thus

$$
\begin{aligned}
& (-1)^{M / 2} \prod_{0<k_{+}<\pi} \sin k_{+} / 2 a_{+}^{*}=2^{-M / 2} \\
& (-1)^{M / 2+1} \prod_{0<k_{-}<\pi} \sin k_{-} / 2 a_{-}^{*}=M 2^{-M / 2}
\end{aligned}
$$

and, of course, $\left|a_{ \pm}\right|=\mid$from (3.20). Hence

$$
a_{+}^{*}=-a_{-}^{*}=(-1)^{M / 2}
$$


from which

$$
| \pm\rangle=(-1)^{M / 2} 2^{-1 / 2}\left(\left|\Phi_{+}^{0}\right\rangle \mp\left|\Phi_{-}^{0}\right\rangle\right)
$$

follows.

The block rotation $R(s)$ on $|-\rangle$ may be written:

$$
\begin{aligned}
R(s) & =\sigma_{1}^{y} \sigma_{s}^{y} \prod_{2}^{s-1}-\sigma_{j}^{z} \\
& =(-1)^{s+1}\left(f_{1}^{\dagger}+f_{1}\right)\left(f_{s}^{\dagger}-f_{s}\right)
\end{aligned}
$$

using Section 2 of Ref. [19]. Evidently $[P, R(s)]_{-}=0$ so (for $M$ even):

$$
Z_{\bar{M}}^{ \pm}(s)=\sum_{\varepsilon=+,-}\left\langle\Phi_{\varepsilon}^{0}\left|R(s) V_{\varepsilon}^{2 N} R(s)\right| \Phi_{\varepsilon}^{0}\right\rangle / 2
$$

to which the method of Ref. [20] may be applied and (for $M$ even)

$$
\Sigma_{M}^{+-}(s \mid p)=\operatorname{Re}\left\langle\Phi_{-}^{0}\left|R(s) V^{N} \sigma_{p}^{x} V_{+}^{N} R(s)\right| \Phi_{+}^{0}\right\rangle .
$$

Spectral decomposition may now be applied to in (3.27) and (3.29). First consider the limiting matrix element:

$$
\lim _{M \rightarrow \infty}\left\langle\Phi_{ \pm}^{0}|R(s)| \Phi_{ \pm}\right\rangle /\left\langle\Phi_{ \pm}^{0} \mid \Phi\right\rangle=\frac{(-1)^{s}}{\pi} \int_{0}^{2 \pi} d \omega \frac{e^{i s \omega}}{1+\cos \delta^{*}(\omega)}
$$

obtained using (3.22), (3.23), (3.27) and the standard method of [20]; the angle $\delta^{*}(\omega)$ is given by:

$$
e^{i \delta^{*}(\omega)}=\left(\frac{B}{A}\right)^{1 / 2}\left[\frac{(z-A)\left(z-B^{-1}\right)}{\left(z-A^{-1}\right)(z-B)}\right]^{1 / 2}
$$

with

$$
\begin{aligned}
& A=\exp 2 K \operatorname{coth} K \\
& B=\exp 2 K \tanh K
\end{aligned}
$$

and $\delta^{*}(0)=0$ selects the branch for $A>B>1$.

It is clear from the Riemann-Lebesgue lemma that such terms vanish in (3.35). The first term which does not vanish, and in fact dominates, comes from $\mathscr{B}_{2}^{ \pm}$; it is:

$$
\begin{aligned}
& \left\langle\Phi_{+}^{0}\left|R(s) G_{-\beta_{1}}^{\dagger} G_{-\beta_{2}}^{\dagger}\right| \Phi_{+}\right\rangle /\left\langle\Phi_{+}^{0} \mid \Phi_{+}\right\rangle \\
& \left.=e^{i\left(\beta_{1}+\beta_{2}\right) / 2}\left(e^{-i s \beta_{1}}-e^{-i s \beta_{2}}\right) / \cos \left(\delta^{*}\left(\beta_{1}\right) / 2\right) \cos \delta^{*}\left(\beta_{2}\right) / 2\right) .
\end{aligned}
$$

Let the term in $\Sigma_{M}^{+-}(s \mid p)$ which arises from $\mathscr{B}_{2}^{ \pm}$be denoted by $\operatorname{Re} A_{M}^{+-}(s \mid p)$ : then

$$
\begin{aligned}
\lim _{M \rightarrow \infty} A_{M}^{+-}(s \mid p) /\left\langle\Phi_{-}^{0} \mid \Phi_{-}\right\rangle\left\langle\Phi_{+}^{0} \mid \Phi_{+}\right\rangle \\
=\frac{1}{4} \frac{1}{(2 \pi)^{4}} \int_{0}^{2 \pi} \ldots \int d(\omega)_{4} \prod_{1}^{4} B\left(\omega_{j}\right)\left(e^{i s \omega_{1}}-e^{i s \omega_{2}}\right)\left(e^{i s \omega_{3}}-e^{i s \omega_{4}}\right) \\
\quad F_{x}\left(e^{i \omega_{1}} e^{i \omega_{2}} \mid e^{i \omega_{3}} e^{i \omega_{4}}\right)
\end{aligned}
$$

where

$$
B(\omega)=e^{i(p-1) \omega} e^{-i \theta(\omega)} e^{-N \gamma(\omega)} / \cos \left(\delta^{*}(\omega) / 2\right) .
$$


In Ref. [19] [see Eq. (4.5)], $F_{x}(\cdot \mid \cdot)$ is defined and evaluated; it has the structure:

$$
\begin{aligned}
& F_{x}\left(z_{1} z_{2} \mid t_{3} t_{4}\right)=m^{*}\left(-f_{-}\left(z_{1} z_{2}\right) f_{-}\left(t_{3} t_{4}\right)\right. \\
& \left.+f_{+}\left(z_{1} t_{3}\right) f_{+}\left(z_{2} t_{4}\right)-f_{+}\left(z_{1} t_{4}\right) f_{+}\left(z_{2} t_{3}\right)\right)
\end{aligned}
$$

where the contraction functions are given by:

$$
f_{ \pm}(z, t)=\left(\frac{B}{A}\right)^{1 / 2} \frac{z t}{z t-1}\left(\left[\frac{(z-A)\left(t-B^{-1}\right)}{(z-B)\left(t-A^{-1}\right)}\right]^{1 / 2}-[t, z]^{1 / 2}\right)
$$

and $m^{*}$ is the spontaneous magnetization.

By using the symmetry evident in (3.33) and (3.35) it follows that:

$$
\begin{aligned}
\lim _{M \rightarrow \infty} A^{+-}(s \mid p) /\left\langle\Phi_{-}^{0} \mid \Phi_{-}\right\rangle\left\langle\Phi_{+}^{0} \mid \Phi_{+}\right\rangle=B(s \mid p) \\
=\frac{\mathrm{P}}{(2 \pi)^{4}} \int_{0}^{2 \pi} \ldots \int d(\omega)_{4} e^{i s\left(\omega_{1}+\omega_{3}\right)} \prod_{1}^{4} B\left(\omega_{j}\right) \\
F^{x}\left(e^{i \omega_{1}} e^{i \omega_{2}} \mid e^{i \omega_{3}} e^{i \omega_{4}}\right)
\end{aligned}
$$

and a similar analysis of the $\mathscr{B}_{2}^{ \pm}$contribution to $Z_{M}^{+-}(s)$, denoted $\left(Z_{M}^{+-}(s)\right)_{2}$, gives:

$$
\begin{aligned}
& \lim _{M \rightarrow \infty}\left(Z_{M}^{+-}(s)\right)_{2} /\left|\left\langle\Phi_{+}^{0} \mid \Phi_{+}\right\rangle\right|^{2}=C(s \mid p) \\
& =\left(\frac{1}{2 \pi}\right)^{2} \int_{0}^{2 \pi} d(\omega)_{2} e^{-2 N\left(\gamma\left(\omega_{1}\right)+\gamma\left(\omega_{2}\right)\right)} \frac{2-e^{i s\left(\omega_{1}-\omega_{2}\right)}-e^{i s\left(\omega_{2}-\omega_{1}\right)}}{\left(1+\cos \delta^{*}\left(\omega_{1}\right)\right)\left(1+\cos \delta^{*}\left(\omega_{2}\right)\right)} .
\end{aligned}
$$

The only term in (3.37) which survives as $s \rightarrow \infty$ contains $f_{+}\left(e^{i \omega_{1}}, e^{i \omega_{3}}\right)$ is the expansion of $F^{x}(\cdot \mid \cdot)$. This gives the final result:

$$
B(s \mid p)=m^{*} I_{N}(2 \pi)^{-2} \int_{0}^{2 \pi} d(\omega)_{2} B\left(\omega_{1}\right) B\left(\omega_{2}\right) f_{+}\left(e^{i \omega_{1}}, e^{i \omega_{2}}\right)
$$

where

$$
I_{N}=\frac{1}{\pi} \int_{0}^{2 \pi} d \omega e^{-2 N \gamma(\omega)} /\left(1+\cos \delta^{*}(\omega)\right)
$$

and

$$
C(s \mid p)=I_{N}^{2}
$$

The manifestation of clustering is obvious, and the final result is:

$$
\left\langle\sigma_{p}\right\rangle_{N} \sim m^{*} I_{N}^{-1} \mathrm{P} \iint_{0}^{2 \pi} d(\omega)_{2} B\left(\omega_{1}\right) B\left(\omega_{2}\right) f_{+}\left(e^{i \omega_{1}}, e^{i \omega_{2}}\right)
$$

as $N \rightarrow \infty$. The remainder of the proof of Theorem 2 is given in the appendices.

\section{Appendix A: Proof of Lemma 1}

First the spectrum of the transfer matrix $\hat{V}$ with + and - edges will be described. With infinite edge weight between rows at the boundaries, we have [7]:

$$
\hat{V}=\exp -\frac{1}{2} \sum_{0}^{2 N} \gamma_{j}\left(2 X_{j}^{\dagger} X_{j}-1\right)
$$



where the $X_{j}$ are Fermi operators, and $\gamma_{0}=0$. Using the notation $X_{I}=\prod_{i \in I} X_{i}$
with index sets $I$ which exclude 0 , we have the spectra:

\begin{tabular}{|c|c|c|}
\hline $\begin{array}{l}\text { Boundary } \\
\text { condition }\end{array}$ & Eigenvectors & $\begin{array}{l}\text { Maximum } \\
\text { eigenvalue }\end{array}$ \\
\hline++ & $\frac{1}{2}\left(1+(-1)^{|I|}\right) X_{I}^{\dagger} X_{0}^{+}|\Phi\rangle$ & $\Lambda_{0}, T<T_{c}$ \\
\hline- & $\frac{1}{2}\left(1+(-1)^{|I|}\right) X_{I}^{\dagger} X_{0}^{-}|\Phi\rangle$ & $\Lambda_{0}, T>T_{c}$ \\
\hline-+ & $\frac{1}{2}\left(1-(-1)^{|I|}\right) X_{I}^{\dagger} X_{0}^{-}|\Phi\rangle$ & $\Lambda_{0} e^{-\gamma(0)}, T<T_{c}$ \\
\hline+- & $\frac{1}{2}\left(1-(-1)^{|I|}\right) X_{I}^{\dagger} X_{0}^{+}|\Phi\rangle$ & $\Lambda_{0}\left(1-O e^{-2 N \gamma(0)}\right), T>T_{c}$ \\
\hline
\end{tabular}

where

$$
X_{i}|\Phi\rangle=0, i=0, \ldots 2 N, X_{0}^{ \pm}=2^{-1 / 2}\left(1 \pm\left(X_{0}^{\dagger}+X_{0}\right)\right) .
$$

The ++ and -- maximal eigenvectors are degenerate, as expected by symmetry. For $T>T_{c}$, there is a $\gamma_{j}$ which is asymptotic to zero as $N \rightarrow \infty$, giving an asymptotic degeneracy of $\hat{V}$; but no such asymptotic degeneracy obtains for $T<T_{c}$, in contrast with the usual case [20]. This is because there is a surface tension associated with a long contour on a cylinder with + on one face and - on the other [3]. The reader may be amused to construct and interpret the rest of the spectrum of $\hat{V}$ for the various boundary conditions [21]. It is also of interest to note that the eigenvalue problems for $\hat{V}$ and $V^{1}=V_{1}^{1 / 2} V_{2} V_{1}^{1 / 2}$ with free edges are related by duality, a result suggested by the work of Gallavotti et al. [4] on the spontaneous magnetization $m^{*}$.

With the notation:

$$
\hat{V}_{1}=\exp -K_{2}^{*} \sum_{1}^{M} \sigma_{j}^{z} \quad \hat{V}_{2}=\exp K_{1} \sum_{1}^{M} \sigma_{j}^{x} \sigma_{j+1}^{x}
$$

the standard transfer matric techniques give:

$$
\begin{aligned}
& \left\langle\sigma_{p}\right\rangle_{\mathscr{O}_{A}{ }^{+-}}=\left(\left\langle-\left|\left(\hat{V}_{2} \hat{V}_{1}\right)^{M} \sigma_{-N}^{z} \sigma_{N}^{z}\left(\hat{V}_{2} \hat{V}_{1}\right)^{M} \hat{V}_{2}\right|+\right\rangle\right)^{-1} \\
& \left\langle-\left|\left(\hat{V}_{2} \hat{V}_{1}\right)^{M} \sigma_{-N}^{z} \sigma_{N}^{z}\left(\hat{V}_{2} \hat{V}_{1}\right)^{p} \sigma_{0}^{x}\left(\hat{V}_{2} \hat{V}_{1}\right)^{M-p} \hat{V}_{2}\right|+\right\rangle .
\end{aligned}
$$

The limit $M \rightarrow \infty$ is easily taken if $T \neq T_{c}$, for then $\gamma_{j}>0 \forall j \neq 0$, and

$$
\begin{aligned}
& \lim _{M \rightarrow \infty}\left\langle\sigma_{p}\right\rangle_{\mathscr{B}_{A}^{+-}} \\
& =\left\langle\Phi\left|X_{0}^{-} \sigma_{-N}^{z} \sigma_{N}^{z}\left(\frac{\hat{V}}{\Lambda_{0}}\right)^{p} \hat{\sigma}_{0} X_{0}^{+}\right| \Phi\right\rangle /\left\langle\Phi\left|X_{0}^{-} \sigma_{-N}^{z} \sigma_{N}^{z} X_{0}^{+}\right| \Phi\right\rangle
\end{aligned}
$$

where $\hat{\sigma}_{0}=\hat{V}_{1}^{1 / 2} \sigma_{0}^{x} \hat{V}_{1}^{-1 / 2}$.

We can set up an analogous argument for the boundary condition $\mathscr{B}_{A}(s)$ on the cylinder: evidently:

$$
\begin{aligned}
\lim _{M \rightarrow \infty}\left\langle\sigma_{p}\right\rangle_{\mathscr{B}_{\Lambda}(s)}= & \left\langle\Phi\left|X_{0}^{-} \sigma_{-N}^{z} \sigma_{N}^{z} \hat{V}^{p} \hat{\sigma}_{0} \hat{V}^{s-p} \sigma_{-N}^{z} \sigma_{N}^{z} X_{0}^{-}\right| \Phi\right\rangle / \\
& \left\langle\Phi\left|X_{0}^{-} \sigma_{-N}^{z} \sigma_{N}^{z} \hat{V}^{s} \sigma_{-N}^{z} \sigma_{N}^{z} X_{0}^{-}\right| \Phi\right\rangle
\end{aligned}
$$

and by repeating the argument as $s \rightarrow \infty$ we have:

$$
\left\langle\sigma_{p}\right\rangle_{N}=\lim _{M \rightarrow \infty}\left\langle\sigma_{p}\right\rangle_{\mathscr{B}_{A}{ }^{+-}}
$$

Note that we have also proved the existence of the limit as $M \rightarrow \infty$ ! 


\section{Appendix B: Asymptotics}

Here we analyse the integral

$$
I(N, p)=\frac{\mathrm{P}}{2 \pi} \iint_{0}^{2 \pi} d(\omega)_{2} B\left(\omega_{1}\right) B\left(\omega_{2}\right) f_{+}\left(e^{i \omega_{1}}, e^{i \omega_{2}}\right)
$$

for large $N$. By using Lemma 2 it suffices, as will be seen, to consider the case:

$$
p=\alpha N^{\delta}
$$

with $\delta<1$. The saddle point in (B.1) is at $\omega_{1}=\omega_{2}=\omega_{0}$ where

$$
\gamma^{(1)}\left(\omega_{0}\right)=i p / N
$$

for which we have:

$$
\omega_{0} \sim i p / N \gamma^{(2)}(0)
$$

as $N \rightarrow \infty$. The path of integration is deformed onto the steepest descent contour $C$ for each variable. By the Plemelj formula, we have:

$$
I(N, p)=\operatorname{sgn} p \int_{0}^{2 \pi} d \omega e^{-2 N \gamma(\omega)}\left(1+\cos \delta^{*}(\omega)\right)^{-1}+I(C)
$$

where

$$
I(C) \sim e^{-\left(2 N \gamma(0)+p^{2} / N \gamma^{(2)}(0)\right)} \frac{1}{\pi} \iint_{C} \frac{a x d y e^{-N \gamma^{(2)}(0)\left(x^{2}+y^{2}\right) / 2}}{i\left(2 \omega_{0}+x+y\right)} .
$$

Now rotating the domain of integration through $\pi / 4$ gives the desired result:

$$
I(C) \sim\left(\pi / N \gamma^{(2)}(0)\right)^{1 / 2} \exp -\left(2 N \gamma(0)+p^{2} / N \gamma^{(2)}(0)\right) J\left(\left(p / N \gamma^{(2)}(0)\right)^{1 / 2}\right)
$$

where

$$
J(x)=\frac{1}{\pi i} \int_{-\infty}^{\infty} \frac{e^{-t^{2}}}{t+i x} d t .
$$

Note that $\lim _{x \rightarrow 0+} J(x)=-1$ and that $J(x)$ is monotone inreasing with $J(\infty)=0$. These results lead directly from (4.42) to Theorem 2.

\section{Appendix C}

Here the matrix elements $\left\langle\Phi_{+}^{0}\left|R(s) G_{\beta_{1}}^{\dagger} G_{\beta_{2}}^{\dagger}\right| \Phi_{+}\right\rangle$will be calculated using the method of Schultz et al. [20]. From (3.27) $R(s)$ is given by:

$$
R(s)=(-1)^{s+1} M^{-1} \sum_{\beta_{3} \beta_{4}} e^{-i \beta_{3}} e^{-i s \beta_{4}}\left(F_{\beta_{3}}^{\dagger}+F_{-\beta_{3}}\right)\left(F_{\beta_{4}}^{\dagger}-F_{-\beta_{4}}\right) .
$$

The trick is to use the structure of $\left|\Phi_{+}\right\rangle$and $\left|\Phi_{+}^{0}\right\rangle$ in which for each $\beta>00$ either $\beta$ and $-\beta$ are both "occupied" or both are "empty". Thus we have an isomorphism in each subspace of this type with a Pauli algebra, which greatly facilitates the working. The result is (3.32), which has the proper antisymmetry in $\beta_{1}$ and $\beta_{2}$. 
It is also easy to evaluate the matrix element

$$
\left\langle\Phi_{+}^{0}\left|R(s) V^{2 N} R(s)\right| \Phi_{+}^{0}\right\rangle
$$

by the same procedure. Referring to (C.1) the terms with internal pairing in $R(s)$ vanish as $s \rightarrow \infty$ after $M \rightarrow \infty$ by the Riemann Lebesgue lemma. Thus the $\beta$ must be paired between different $R(s)$ factors.

Consequently $V^{2 N}$ works in the subspace for $\beta>0$ spanned by $F_{-\beta}^{+}|0\rangle$ and $F_{\beta}^{+}|0\rangle$ in which it acts as the identity. Thus we have immediately that:

$$
\begin{aligned}
\left\langle\Phi_{+}^{0}\left|R(s) V^{2 N} R(s)\right| \Phi_{+}^{0}\right\rangle \\
=\frac{P}{M} \sum_{>0}^{<\pi}\left(\cosh 2 N \gamma(\beta)+\sinh 2 N \gamma(\beta) \cos \delta^{*}(\beta)\right)^{-1} \\
+ \text { (term which vanishes in limit as } s \rightarrow \infty) . \\
\text { Here } \\
P=\prod_{>0}^{<\pi}\left(\cosh 2 N \gamma(\beta)+\sinh 2 N \gamma(\beta) \cos \delta^{*}(\beta)\right) \\
=\left\langle\Phi_{+}^{0}\left|V^{2 N}\right| \Phi_{+}^{0}\right\rangle .
\end{aligned}
$$

The desired result is:

$$
\lim _{s \rightarrow \infty} \lim _{M \rightarrow \infty} \frac{\left\langle\Phi_{ \pm}^{0}\left|R(s) V^{2 N} R(s)\right| \Phi_{ \pm}^{0}\right\rangle}{\left\langle\Phi_{ \pm}^{0}\left|V^{2 N}\right| \Phi_{ \pm}^{0}\right\rangle}=J_{N}^{2}
$$

where

$$
J_{N}=\frac{1}{2 \pi} \int_{0}^{2 \pi} d \omega\left(\cosh 2 N \gamma(\omega)+\sinh 2 N \gamma(\omega) \cos \delta^{*}(\omega)\right)^{-1} .
$$

Thus the error term is immediately computed:

$$
\begin{aligned}
& \left.\left.\lim _{s \rightarrow \infty} \lim _{M \rightarrow \infty} \sum_{\varepsilon}\left(\left\langle\Phi_{\varepsilon}^{0}\left|R(s) V_{\varepsilon}^{2 N} R(s)\right| \Phi_{\varepsilon}^{0}\right\rangle-\sum_{j \in \mathscr{B}_{0}^{\varepsilon} \cup \mathscr{B}_{2}^{\varepsilon}} K \Phi_{\varepsilon}^{0}|R(s)| j\right\rangle\right|^{2} \Lambda_{j}^{2 N}\right) / \\
& \sum_{\varepsilon}\left\langle\Phi_{\varepsilon}^{0}\left|V_{\varepsilon}^{2 N}\right| \Phi_{\varepsilon}^{0}\right\rangle \\
& =\frac{1}{\pi} \int_{0}^{2 \pi} d \omega \frac{e^{-2 N \gamma(\omega)}}{1+\cos \delta^{*}(\omega)}\left[\left(1+e^{-4 N \gamma(\omega)} \frac{1-\cos \delta^{*}}{1+\cos \delta^{*}}\right)^{-1}-1\right] .
\end{aligned}
$$

Finally, note (3.18).

Acknowledgements. The firstnamed author would like to thank C. J. Preston, M. E. Fisher, J. L. Lebowitz, A. Martin-Löf, O. Penrose, and B. Widom for discussions on various aspects of this work, and especially G. Gallavotti for enlightenment and encouragement; he acknowledges the generous support of the Ecole Polytechnique Fédérale of Lausanne, while on leave from Oxford University, the kind hospitality of $\mathrm{H}$. Kunz and $\mathrm{Ph}$. Choquard and the stimulating atmosphere of the "Semaine de Mécanique Statistique» organized by the 3e Cycle de la Physique En Suisse Romande, Pully, Switzerland. 


\section{References}

0. Result first reparted by Abraham, D. B. and Reed, P. Phys. Rev. Letters 33, 377 (1974)

1. Gallavotti, G.: Riv. Nuovo Cimento 2, 133 (1972), Gallavotti,G., Martin-Löf,A., MiracleSole, S.: In: Statistical Mechanics and Mathematical Problems (ed. A. Lenard, p. 162). BerlinHeidelberg-New York: Springer 1973

2. Camp, W.J., Fisher, M.E.: Phys. Rev. B6, 946 (1972), and ref. 19 cited therein;

Minlos, R. A., Sinai, J. G. : Mat. USSR Sbornik 2, 395 (1967); English translation: Trans. Moscow Math. Soc. 19, 121 (1968)

3. Abraham, D. B., Gallavotti, G., Martin-Löf, A.: Physica (Utrecht) 65, 73 (1973)

4. Onsager, L.: Nuovo Cimento (Suppl.) 6, 261 (1949)

Yang, C. N.: Phys. Rev. 85, 808 (1952);

Bennettin, G., Gallavotti, G., Jona-Lasinio, G., Stella, A.L.: Commun. math. Phys. 30, 45 (1973), see also Ref. 7

5. Messager, A., Miracle-Sole, S.: Commun. math. Phys. 40, 187 (1975);

Gallavotti, G., Miracle-Sole, S.: Phys. Rev. B5, 2555 (1972)

6. Martin-Löf, A.: Commun. math. Phys. 24, 253 (1972);

Lebowitz, J.L., Martin-Löf, A.: Commun. math. Phys. 25, 276 (1972)

Ruelle,D.: Ann. Phys. (New York) 69, 364 (1972)

Dobrushin, R.L.: Teorya Veroyatnostlii ill Primeneniya 13, 201 (1968)

Lanford,O.E., Ruelle, D.: Commun. math. Phys. 13, 194 (1969)

7. Abraham, D. B., Martin-Löf, A.: Commun. math. Phys. 32, 245 (1973)

8. Onsager,L.: Phys. Rev. 65, 117 (1944)

9. Fisher, M.E., Ferdinand, A.E.: Phys. Rev. Letters 26, 565 (1971)

10. Gallavotti, G., Martin-Löf, A.: Commun. math. Phys. 25, 87 (1972)

11. Gallavotti, G.: Commun. math. Phys. 27, 103 (1972)

12. Messager,A.: Semaine de Mécanique Statistique, organisée par le 3e cycle de la physique en Suisse Romande, Cully, canton de Vaud

13. Dobrushin, R. L.: Theory Prob. Appl. 17, 582 (1972)

14. Van Beijeren, H.: Commun. math. Phys. 40, 1 (1975)

15. Weeks, J.D., Gilmer, G. H., Leamy: Phys. Rev. Letters 31, 549 (1973)

16. Temperley, H. N. V.: Proc. Camb. Phil. Soc. 48, 683 (1952)

17. See Minlos, R. A., Sinai, J. G.: Ref. 2

18. Fortuin, C. M., Kasteleyn, P.W., Ginibre, J.: Commun. math. Phys. 22, 89 (1971)

19. Abraham, D. B.: To be published

20. Schultz,T.D., Matthis, D.C., Lieb,E.H.: Rev. Mod. Phys. 36, 856 (1964)

21. Abraham, D. B.: Studies Appl. Math. 51, 179 (1972)

22. Fisk, S., Widom, B.: J. Chem. Phys. 50, 3219 (1969)

See also the review by Widom, B., in Phase Transitions and Critical Phenomena, Vol. II (eds. C.Domb, M.S.Green). London: Academic Press 19

Communicated by G. Gallavotti

Received December 15, 1975 